\title{
REÚSO EN PROCESOS DE BLANQUEO TEXTIL DE LOS EFLUENTES PROVENIENTES DE TINTURAS CON COLORANTES REACTIVOS NEGROS DECOLORADOS MEDIANTE EL MÉTODO DE FENTON
}

\author{
Carmen L. Uribe Valenzuela ${ }^{* a}$, Melissa J. Caballero Bustos ${ }^{\mathrm{a}}$, \\ Percy O. Cárdenas Torres ${ }^{b}$, Emilia G. Hermoza Guerra ${ }^{b}$
}

\begin{abstract}
RESUMEN
Se reusaron efluentes textiles provenientes de teñidos negros reactivos, decolorados por el método de oxidación avanzada Fenton en procesos de blanqueo de fibra celulósica por agotamiento. Se recolectaron efluentes textiles provenientes de dos teñidos reactivos negros: Corafix Black SD y Corazol Black B 133 \%, simulados y reales, para ser decolorados con el tratamiento Fenton. Se monitoreó la decoloración usando espectrofotometría UV-visible, se determinaron las proporciones óptimas de reactivo Fenton (sal de Fe (II) $(\mathrm{g}) / \mathrm{H}_{2} \mathrm{O}_{2}(\mathrm{~mL})$ ): $0,0156 \mathrm{~g} / \mathrm{mL}$ y $0,0251 \mathrm{~g} / \mathrm{mL}$ con un tiempo de reacción de 90 minutos y $\mathrm{pH}$ óptimos de tratamiento de 3,70 y 3,88 para los efluentes textiles provenientes de teñido con colorantes Corafix Black SD y Corazol Black B 133\%, respectivamente. Los efluentes tratados se caracterizaron, adecuaron y usaron en el blanqueo de tejidos celulósicos. Se obtuvo textiles con un grado de blancura de $74,73{ }^{\circ} \mathrm{Be}$ y $73,41^{\circ} \mathrm{Be}$, similares al sustrato blanqueado con agua blanda $\left(76,61^{\circ}\right.$ Berger). Se compararon: las curvas de reflectancia con el espectrofotómetro Datacolor SF550, hidrofilidad y capilaridad según métodos normalizados. Finalmente se concluyó que el tratamiento Fenton es viable para este tipo de efluentes negros reactivos, pues se obtuvieron sustratos blanqueados con propiedades similares al blanqueo con agua blanda.
\end{abstract}

Palabras clave: efluente textil, Fenton, blanqueo químico, colorante reactivo.

\footnotetext{
a Escuela Profesional de Ingeniería Textil de la Facultad de Ingeniería Química y Textil, Universidad Nacional de Ingeniería, Av. Túpac Amaru 210, Lima 25, Perú, curibe@uni.edu.pe

b Escuela Profesional de Ingeniería Química de la Facultad de Ingeniería Química y Textil, Universidad Nacional de Ingeniería, Av. Túpac Amaru 210, Lima 25, Perú.
} 


\title{
REUSE IN TEXTILE BLEACHING PROCESSES OF EFFLUENTS FROM DYEING WITH REACTIVE BLACK DYES DECOLORED BY THE FENTON METHOD
}

\begin{abstract}
Textile effluents from reactive black dyes, decolorized using the advanced Fenton oxidation method, were reused in cellulosic fiber exhaustion bleaching processes. Textile black reactive effluents were collected, provided from two recipes of reactive blacks; Corafix Black SD (Black SD) and Corazol Black B $133 \%$ (Black 5), simulated and real, due to being decolored by the Fenton treatment. The discoloration was monitored using UVvisible spectrophotometry, the optimal proportions of Fenton reagent (Fe (II) salt (g) / $\mathrm{H}_{2} \mathrm{O}_{2}$ $(\mathrm{mL})$ ) were determined: $0,0156 \mathrm{~g} / \mathrm{mL}$ and $0,0251 \mathrm{~g} / \mathrm{mL}$ with 90 minutes of reaction time, and optimal treatment $\mathrm{pH}$ of 3,70 and 3,88 for textile effluents from dyeing with Corafix Black SD and Corazol Black B $133 \%$ dyestuffs respectively. The discolored effluents were characterized, adequated, and used in bleaching of cellulosic fabrics. Textiles with a degree of whiteness of $74,73{ }^{\circ} \mathrm{Be}$ and $73,41^{\circ} \mathrm{Be}$ were obtained, similar to the substrate bleached with soft water $\left(76,61^{\circ} \mathrm{Be}\right)$. The reflectance curves were compared with the Datacolor SF550 spectrophotometer, hydrophilicity and capillarity according to standardized methods. Finally, it was concluded that the Fenton treatment is viable for this type of reactive black effluent since bleached substrates with properties similar to bleaching with soft water were obtained.
\end{abstract}

Key words: textile effluent, Fenton, bleaching, reactive dyestuff.

\section{INTRODUCCIÓN}

La industria textil es uno de los sectores productivos de mayor consumo de agua, consumiendo desde 30 a $150 \mathrm{~L} / \mathrm{kg}$ material textil acabado ${ }^{1}$. Actualmente, las normativas medioambientales, la escasez y el encarecimiento del recurso hídrico obligan al ahorro de agua en los procesos textiles. Las tintorerías textiles aparte de consumir altos volúmenes de agua, generan efluentes con distintas concentraciones de colorantes y químicos, los cuales ocasionan impactos negativos al ambiente si no son gestionados adecuadamente antes de verterse al alcantarillado y posteriormente a los ríos y océanos.

Los colorantes reactivos son los más usados para el teñido del algodón y generan efluentes problemáticos debido al bajo poder de agotamiento y fijación en la fibra. El colorante no fijado se encuentra en altas concentraciones en los efluentes textiles ${ }^{2}$, por consiguiente, es un efluente no sólo difícil de tratar sino también de reusar.

Los procesos de oxidación avanzada son aquellos tratamientos terciarios que usan oxidantes (químicos) para reducir los niveles DQO/DBO, y para separar los componentes orgánicos 
y los componentes inorgánicos oxidables ${ }^{3}$. El método Fenton es uno de los tratamientos de oxidación avanzada que ha mostrado buenos resultados para la decoloración de colorantes azoicos ${ }^{4}$.

Se seleccionó el método Fenton para la decoloración ya que es uno de los procesos más económicos y efectivos, que consiste en la adición de sales de hierro en medio ácido para promover la descomposición catalítica del peróxido de hidrógeno en radicales hidroxilo $\mathrm{HO}$, los cuales son capaces de oxidar la materia orgánica y los compuestos inorgánicos, tales como sulfuros presentes en el agua residual ${ }^{5}$.

Entre los procesos textiles de mayor importancia se encuentra el ennoblecimiento textil que abarca: teñido, estampados y acabados. Entre los procesos previos al teñido tenemos uno de los más empleados en la industria textil: el blanqueo con peróxido de hidrógeno, que tiene por objetivo remover las impurezas, pigmentos naturales del sustrato y conferir una hidrofilidad que le permita al textil ser teñido sin inconvenientes. El enfoque del proyecto se basó en este proceso ya que es uno de los procesos fundamentales para cualquier tratamiento de teñido y/o ennoblecimiento textil.

\section{PARTE EXPERIMENTAL}

La parte experimental fue realizada en los laboratorios de las Escuelas Profesionales de Ingeniería Química e Ingeniería Textil de la Universidad Nacional de Ingeniería, debido al enfoque multidisciplinario de la investigación esta fue estructurada en dos etapas.

\section{Primera etapa: Decoloración del efluente textil}

Consistió en el tratamiento químico de los efluentes textiles, considerando como variable la dosificación del reactivo Fenton $\left(\mathrm{Fe}^{2+} / \mathrm{H}_{2} \mathrm{O}_{2}\right)$, y como parámetros fijos la velocidad de agitación (rpm) y la temperatura. Las características de los efluentes textiles reales (ER) provenientes del proceso de teñido con los colorantes reactivos Corafix Black SD (Black SD) y Corazol Black B 133\% (Black 5) fueron las siguientes, DQO: $1337,5 \mathrm{mg} / \mathrm{L}$ y $3525,0 \mathrm{mg} / \mathrm{L}$, pH: 11,46 y 12,60, respectivamente. Los espectros UV-vis de ambos efluentes se muestran en la figura 1, fueron obtenidos con un espectrofotómetro UV-visible Shimadzu 1601. 

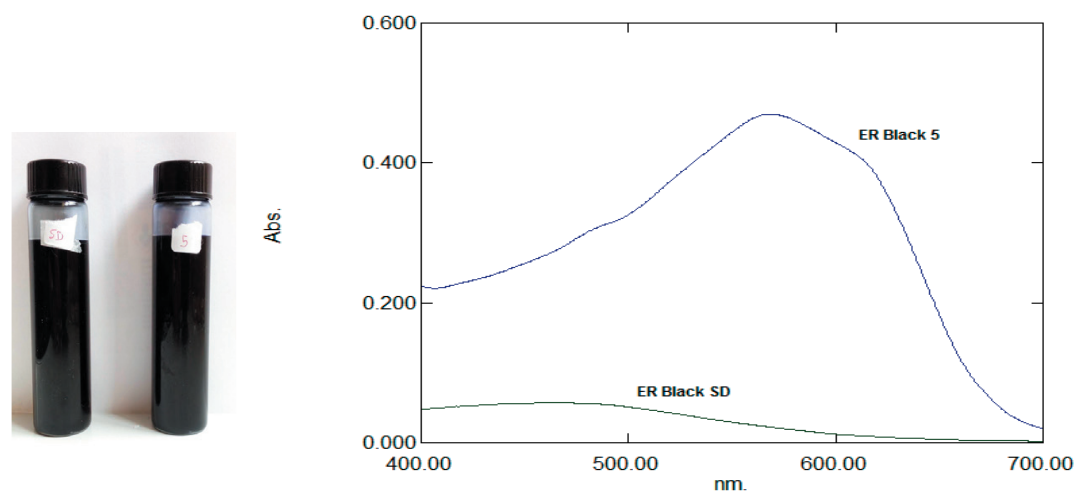

Figura 1. Izquierda. (a) Efluente real del colorante reactivo Corafix Black SD, (b) Efluente real del colorante reactivo Corazol Black B 133 \%. Derecha. Espectros UV-vis de los efluentes reales (ER) de los colorantes Black SD y Black 5 diluidos 2:250.

La decoloración de los colorantes negros reactivos se realizó en medio ácido. La acidificación del efluente real a tratar se hizo con $\mathrm{H}_{2} \mathrm{SO}_{4} 18 \mathrm{M}$. En pruebas preliminares se comprobó la decoloración de los efluentes textiles simulados al aplicar el tratamiento Fenton a $\mathrm{pH}=2,8^{3,4}$. Este $\mathrm{pH}$ sirvió como punto inicial para aplicar este tratamiento a los efluentes reales y ensayar el blanqueo de sustratos textiles con el efluente tratado. El pH fue ajustado hacia valores mayores hasta lograr un grado de blanco aceptable en sustratos textiles, encontrándose los siguientes valores óptimos: $\mathrm{pH}=3,70$ para el colorante Black $\mathrm{SD}$ y $\mathrm{pH}=3,88$ para el colorante Black 5.

La dosificación de reactivo Fenton (peróxido de hidrógeno grado industrial al 50 \% y sulfato ferroso heptahidratado al 99,5\%) se determinó en base al DQO según Rodríguez et al. ${ }^{6}$. El efluente tratado se neutralizó con $\mathrm{NaOH} 9 \mathrm{M}$ a fin de formar y precipitar el hidróxido férrico, el cual fue separado mediante filtración al vacío.

\section{Tratamiento óptimo}

Se escaló hasta $200 \mathrm{~mL}$ de efluente textil real con peróxido de hidrógeno al $50 \%$ de grado industrial, la velocidad de agitación fue de $150 \mathrm{rpm}$ y tiempo de tratamiento de $90 \mathrm{~min}$ en un equipo agitador orbital. En la tabla 1 se muestra la caracterización antes del tratamiento y las tres dosis de reactivo Fenton ensayadas para los dos tipos de efluentes textiles reales con los colorantes reactivos. Para el efluente del colorante Black SD las pruebas preliminares A, B y C se denominaron PP1A, PP1B, PP1C; mientras que para el efluente del colorante Black 5 las pruebas 1, 2 y 3 se denominaron: P1, P2 y P3. 
Tabla 1. Caracterización de los efluentes reales que contienen colorantes reactivos negros antes del tratamiento y dosificación del reactivo Fenton para $200 \mathrm{~mL}$ de efluente.

\begin{tabular}{lccccccc}
\hline & \multicolumn{3}{c}{ Colorante Black SD } & & \multicolumn{3}{c}{ Colorante Black 5 } \\
& PP1A & PP1B & PP1C & & P1 & P2 & P3 \\
\hline pH inicial & 11,46 & 11,46 & 11,46 & & 12,60 & 12,60 & 12,60 \\
Conductividad $(\mathrm{mS})$ & 132,1 & 132,1 & 132,1 & & 116,6 & 116,6 & 116,6 \\
ORP $(\mathrm{mV})$ & $-63,3$ & $-63,3$ & $-63,3$ & & $-75,5$ & $-75,5$ & $-75,5$ \\
$\mathrm{pH}$ acondicionado & 3,70 & 3,70 & 3.70 & & 3,88 & 3,88 & 3,88 \\
Masa de FeSO $4.7 \mathrm{H}_{2} 0(\mathrm{~g})$ & 1,00 & 0,292 & 0,280 & & 0,23 & 0,47 & 0,75 \\
$\mathrm{H}_{2} \mathrm{O}_{2} 50 \% \mathrm{~V} / \mathrm{V}(\mathrm{mL})$ & 20,00 & 18,70 & 14,00 & & 18,70 & 18,70 & 18,70 \\
$\mathrm{Sal} \mathrm{de} \mathrm{Fe}(\mathrm{II})(\mathrm{g}) / \mathrm{H}_{2} \mathrm{O}_{2}(\mathrm{~mL})$ & 0,050 & 0,0156 & 0,020 & & 0,0123 & 0,0251 & 0,0401 \\
\hline
\end{tabular}

\section{Segunda etapa: Reúso}

Se caracterizaron el efluente textil tratado por el tratamiento Fenton y el sustrato textil de algodón crudo. Se definió la receta y la curva de proceso para los ensayos de blanqueos con los efluentes tratados. Finalmente, estos sustratos blanqueados fueron comparados respecto al sustrato blanqueado con agua blanda, denominado blanco estándar, mediante normas y lecturas en el espectrofotómetro de reflectancia.

\section{Caracterización del efluente tratado}

Se determinaron las siguientes características de los efluentes tratados por el tratamiento Fenton: Residuos no volátiles (NVR), $\mathrm{pH}$, dureza (ppm de $\mathrm{Ca}^{2+}$ y ppm de $\mathrm{Mg}^{2+}$ ) para cada uno de los efluentes provenientes de tinturas reactivas con los colorantes Black SD y Black 5. Estas características están ligadas al rendimiento del blanqueo de algodón y su evaluación permite asegurar la viabilidad del proceso de blanqueo a partir de estos efluentes tratados, esto se muestra en la tabla 2.

Tabla 2. Características de los efluentes textiles tratados antes de su aplicación en procesos de blanqueo.

\begin{tabular}{lcccc}
\hline Procedencia del efluente & $\begin{array}{c}\text { Residuos no } \\
\text { volátiles (NVR) }\end{array}$ & $\mathrm{pH}$ & $\begin{array}{c}\text { Dureza } \\
(\mathrm{ppm})\end{array}$ & $\begin{array}{c}\mathrm{Fe} \\
(\mathrm{ppm})\end{array}$ \\
\hline Corazol negro B 133\%(Black 5) & 11,55 & 7,50 & 11,37 & 5,63 \\
Corafix negro SD (Black SD) & 11,10 & 7,32 & 10,53 & 3,77 \\
\hline
\end{tabular}

\section{Caracterización del sustrato textil}

Se seleccionó un sustrato estándar de fibra celulósica: tejido crudo de ligamento jersey, título $30 / 1 \mathrm{Ne}$, de composición $100 \%$ algodón y gramaje $141 \mathrm{~g} / \mathrm{m}^{2}$. En la tabla 3 se muestran las características del tejido crudo que se usó en las pruebas de blanqueo a nivel laboratorio con agua blanda y con el efluente tratado con reactivo Fenton. 
Tabla 3. Características del tejido crudo de algodón antes del proceso de blanqueo.

\begin{tabular}{lll}
\hline Característica & Método de ensayo- Norma & Valor obtenido \\
\hline pH de extracción de la tela & AATCC 81-2012 & 8,14 \\
Hidrofilidad & AATCC 79-2014 & mayor a 1 hora \\
Capilaridad & AATCC 197-2013 & $0 \mathrm{~cm}$ en $30 \mathrm{~min}$ \\
\hline
\end{tabular}

\section{Proceso estándar de blanqueo químico del algodón}

Se determinó la receta del blanqueo de algodón por agotamiento, tabla 4 , la cual fue usada en todos los ensayos.

Tabla 4. Receta del proceso blanqueo químico del algodón por agotamiento.

\begin{tabular}{|c|c|c|}
\hline Producto & Función & Concentración \\
\hline \multicolumn{3}{|c|}{ Blanqueo químico $\left(9^{\circ} \mathrm{C}, 45 \mathrm{~min}\right)$ : } \\
\hline Goldpal DNF-EL & Detergente /Humectante & $1,00 \mathrm{~g} / \mathrm{L}$ \\
\hline Goldwhite SB2 & Estabilizador de $\mathrm{H}_{2} \mathrm{O}_{2}$ & $2,00 \mathrm{~g} / \mathrm{L}$ \\
\hline Peróxido de hidrógeno $50 \%$ & Agente oxidante & $4,00 \%$ \\
\hline Hidróxido de sodio $50^{\circ} \mathrm{Be}$ & Álcali & $2,00 \mathrm{~g} / \mathrm{L}$ \\
\hline \multicolumn{3}{|l|}{ Neutralizado $\left(50^{\circ} \mathrm{C}, 10 \mathrm{~min}\right)$ : } \\
\hline Ácido acético $100 \%$ & Ácido & $1,00 \mathrm{~g} / \mathrm{L}$ \\
\hline \multicolumn{3}{|c|}{ Eliminación de residual de peróxido de hidrógeno $\left(40^{\circ} \mathrm{C}, 10 \mathrm{~min}\right)$ : } \\
\hline Goldgreen BIO-P & Degradación del oxidante & $1,00 \%$ \\
\hline
\end{tabular}

En la figura 2 se detalla la curva estándar del proceso de blanqueo químico para el algodón usado tanto para el blanco estándar como para los blanqueos con los efluentes textiles tratados.

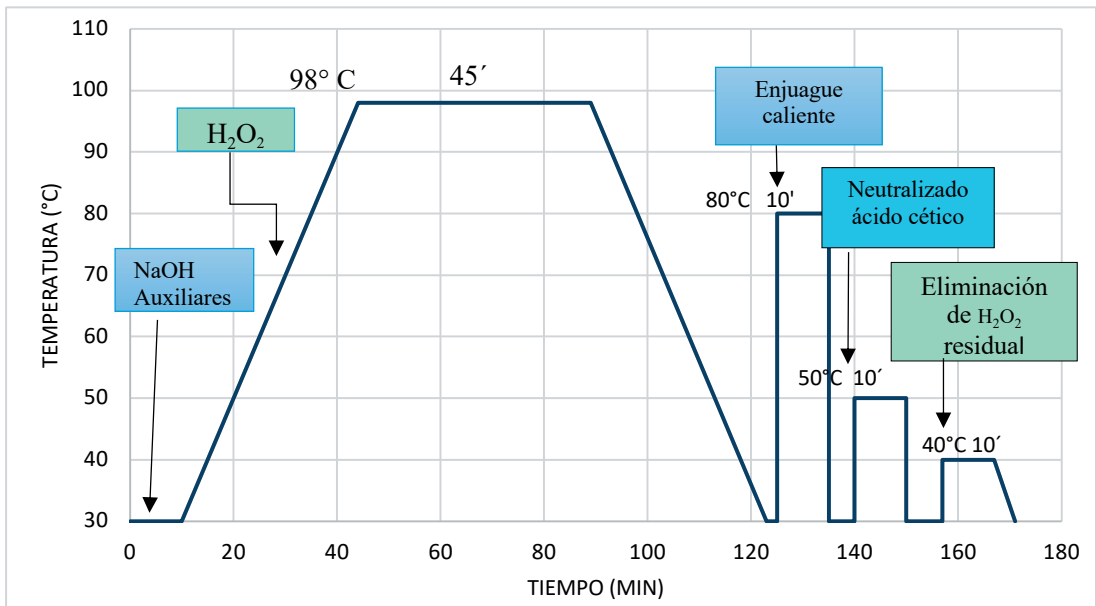

Figura 2. Curva estándar del proceso de blanqueo químico de algodón. 
Se realizaron las pruebas del proceso de blanqueo por agotamiento en la máquina de teñido con agitación vertical con glicerina como medio de calentamiento ubicada en el Laboratorio 24. Posteriormente se evaluó el blanqueo estándar y los blanqueos a partir de los efluentes textiles tratados en la cabina de luces de manera visual bajo luz D65 e instrumentalmente con el espectrofotómetro de reflectancia Datacolor SF 550. Se evaluaron características físicas tales como: igualación, hidrofilidad, capilaridad.

\section{RESULTADOS Y DISCUSIÓN}

\section{Decoloración con método Fenton}

Las pruebas preliminares de decoloración de los efluentes textiles simulados mediante el método Fenton, permitieron establecer un punto de partida para los ensayos de decoloración de los dos efluentes reales de tinturas de celulosa con colorantes reactivos usados en la industria textil: Black SD y Black 5. Al realizar los ensayos de blanqueo con los efluentes reales tratados a $\mathrm{pH}=2,8^{3,4}$ se obtuvo un grado de blanco Berger no satisfactorio en los sustratos celulósicos comparados con el blanco estándar con agua blanda $\left(76,61^{\circ} \mathrm{Be}\right)$. Tomando en cuenta la experiencia de otros autores ${ }^{9,10,13}$ se ajustó el $\mathrm{pH}$ a valores mayores y se encontró los siguientes valores óptimos: $\mathrm{pH}=3,70$ y $\mathrm{pH}=3,88$ para Black $\mathrm{SD}$ y Black 5 respectivamente. En la tabla 5 se listan seis investigaciones con valores de $\mathrm{pH}>2,8$ para tratamientos Fenton aplicados a colorantes reactivos donde se lograron resultados aceptables en la remoción del color y en la reducción de la DQO.

Tabla 5. Colorantes reactivos para celulosa tratados con el reactivo Fenton a distintos valores de $\mathrm{pH}$ óptimos.

\begin{tabular}{lcl}
\hline \multicolumn{1}{c}{ Colorante } & $\mathrm{pH}$ & \multicolumn{1}{c}{ Fuente } \\
& óptimo & \\
\hline Amarillo 160, Azul 81, Rojo 190 & 3,50 & Terán, Posligua \& Banchón, 2015 \\
Reactive Black 5 & $3,0-3,5$ & Meric, Kaptan \& Olmez, 2004 \\
Reactive Black 5 & 4,00 & Ribeiro, Andrade \& Toshio, 2015 \\
Reactive Blue 15 & 3,15 & Clemente, 2008 \\
Reactive Blue, Sunfix Ruby, Yellow & 3,50 & Gutierrez, 2015 \\
Reactive Black 5 & 3,00 & Lucas \& Peres, 2006 \\
\hline
\end{tabular}

Se puede decir que al ser un efluente textil real un sistema multicomponente, el valor recomendado de $\mathrm{pH}=2,8$ para evitar la formación y precipitación del $\mathrm{Fe}(\mathrm{OH})_{3}$ no es necesariamente el mejor, pues pueden hallarse algunos componentes que estabilicen a la especie $\mathrm{Fe}^{3+}$ a pH mayores. En el caso de hierro inmovilizado en films de acetato de celulosa del grupo de Ribeiro ${ }^{10}$ se alcanzó un $\mathrm{pH}=4$ sin precipitación de $\mathrm{Fe}(\mathrm{OH})_{3}$. Realizar el tratamiento Fenton a pH mayores que 2,8 es ventajoso para los casos en estudio porque al llevarlos a la escala industrial significaría un ahorro de reactivos en el proceso de acondicionamiento de los efluentes textiles cuyo $\mathrm{pH}$ inicial es mayor que 11 . 
Se procedió a la neutralización y filtración de los efluentes textiles tratados para retirar las especies de hierro, indeseables en el blanqueo químico de fibra celulósica. Se notó un color amarillo pálido en el efluente real tratado del colorante Black 5, mas no en el colorante Black SD, esto podría estar asociado al mayor contenido de hierro remanente luego de la precipitación por neutralización (ver tabla 2). En la tabla 6 se muestra la caracterización de los efluentes tratados según las condiciones óptimas de $\mathrm{pH}$ y dosificación de reactivo Fenton. Se puede observar que los valores de ORP son positivos lo que está en relación con el poder oxidante del peróxido de hidrógeno remanente.

Tabla 6. Caracterización de los efluentes reales después del tratamiento Fenton (90min), luego de neutralizar y filtrar.

\begin{tabular}{llllllll}
\hline & \multicolumn{3}{c}{ Prueba Black SD } & & \multicolumn{3}{c}{ Prueba Black 5 } \\
\cline { 2 - 3 } \cline { 7 - 8 } & PP1A & PP1B & PP1C & & P1 & P2 & P3 \\
\hline pH final (neutralizado) & 7,32 & 7,32 & 7,32 & & 7,02 & 7,27 & 7,44 \\
Conductividad (mS) & 120,4 & 117,5 & 117,7 & & 118,9 & 123,5 & 126,8 \\
ORP (mV) & 261,6 & 250,8 & 165,6 & & 84,2 & 312,7 & 294,9 \\
\hline
\end{tabular}

También se obtuvieron los espectros UV-visible que se muestran en las figuras 3 y 4 , colorante Black 5 y Black SD, respectivamente. Se apreció una notable disminución de la absorbancia al comparar los espectros UV-vis del colorante Corazol Black B 133 \% (Black 5) antes y después del tratamiento de decoloración (figura 1 derecha y figura 3 ), esto a causa del rompimiento de enlaces por la oxidación de la materia orgánica. El punto óptimo de tratamiento se asignó al de mínima absorbancia, que fue la prueba P2 (figura 3) con una relación de reactivo Fenton (sal de Fe (II) (g) / H2O2 (mL)) de 0,0251 g/mL a un pH acondicionado de 3,88.

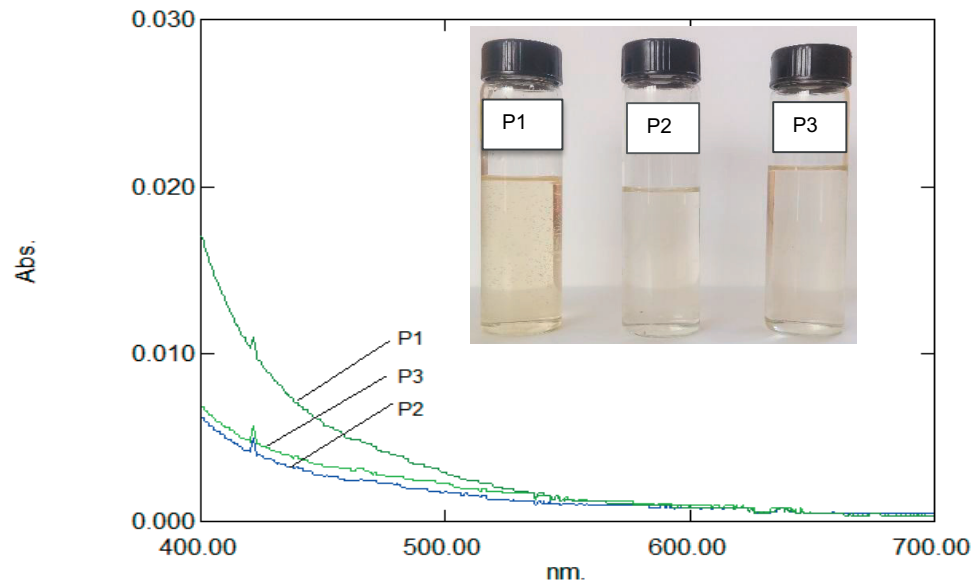

Figura 3. Espectros UV-vis del efluente real después del tratamiento Fenton del colorante Corazol Black B $133 \%$ (Black 5) para las pruebas P1, P2, P3 diluidos 2:25, respectivamente. 
En la figura 4 del análisis del espectro obtenido del colorante Corafix Black SD (Black SD) después del tratamiento de decoloración y en comparación con su espectro inicial (figura 1, derecha), también se observó una disminución apreciable de la absorbancia llegando a valores menores al 0,01 en el rango visible, resultando óptimo el tratamiento con una relación de reactivo Fenton (sal de Fe(II) (g) / H2O2 (mL)) de 0,0156 g/mL, correspondiente a la prueba PP1B a un $\mathrm{pH}$ acondicionado de 3,70.

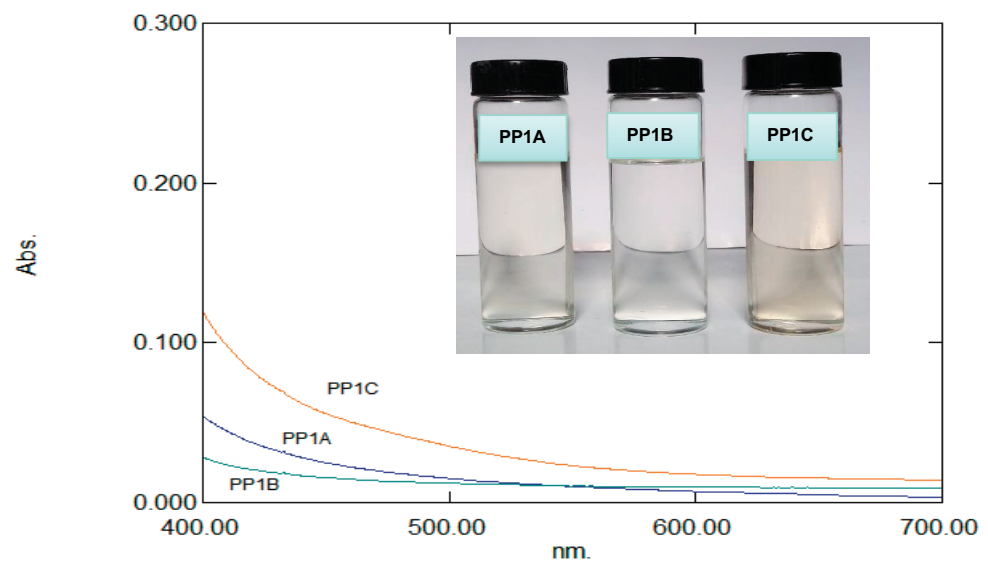

Figura 4. Espectros UV-vis del efluente real después del tratamiento Fenton del colorante Corafix Black SD (Black SD) usando $\mathrm{H} 2 \mathrm{O} 250 \%$ industrial para las pruebas PP1A, PP1B, y PP1C.

Se observa en la zona ultravioleta de las figuras 5 y 6 , un cambio en el perfil del espectro de los efluentes reales tratados respecto a los iniciales y la existencia de un pico remanente después del tratamiento Fenton en las condiciones óptimas. Se infiere que hay una mineralización incompleta, esto debido a que existe la inhibición del Fe(III) con H202, lo que dificulta la formación de $\bullet \mathrm{OH}$, pudiéndose formar complejos de iones de hierro cuando se alcanzan $\mathrm{pH}$ menores a 2,5 al terminar el tratamiento. ${ }^{3,4}$ 


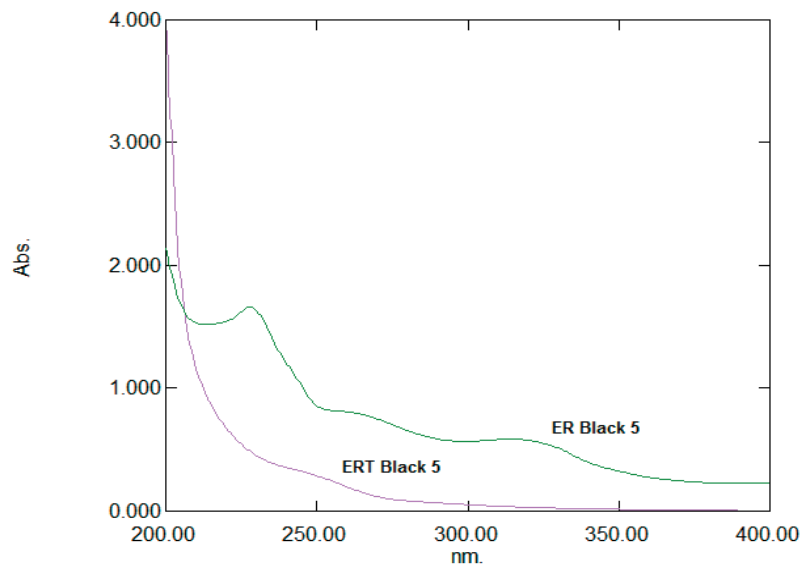

Figura 5. Espectros UV-vis del efluente real antes (ER Black 5 diluido 2:250) y después del tratamiento (ERT Black 5 diluido 2:25) Fenton del colorante Corazol Black B $133 \%$.

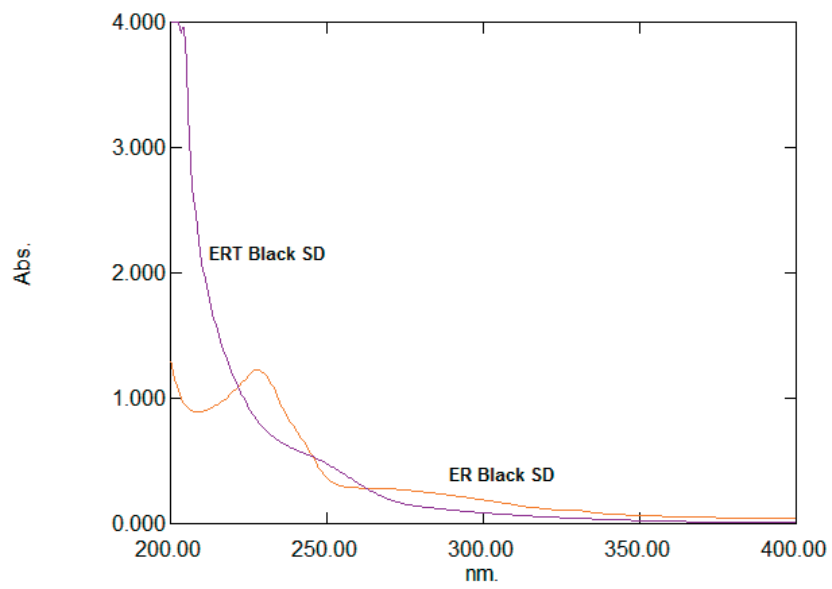

Figura 6. Espectros UV-vis del efluente real antes (ER Black SD diluido 2:250) y después del tratamiento (ERT Black SD diluido 2:25) Fenton del colorante Corafix Black SD.

\section{Reúso de efluentes en procesos de blanqueo químico por agotamiento}

Posterior al proceso de blanqueo químico a partir efluentes reales tratados con diferentes dosis del proceso Fenton se obtuvieron los siguientes resultados para el blanqueo a partir de efluentes decolorados del colorante Black 5 que se muestran en la tabla 7. Las pruebas de blanqueo $\mathrm{Pi}^{\circ} 1$ y $\mathrm{Pi} \mathrm{N}^{\circ} 2$ fueron las pruebas iniciales a partir de un volumen de $50 \mathrm{~mL}$. En el tercer grupo de pruebas se realizó un blanqueo con la dosificación Fenton ajustada de 0,0251 $\mathrm{g} / \mathrm{mL}$, según la tabla 1 , para el colorante Black 5. Para demostrar su reproducibilidad se repitió 3 veces el mismo blanqueo y se evidenció que fue la mejor dosificación al obtener el tejido de algodón con el mejor grado de blanco $\left(73,41^{\circ} \mathrm{Be}\right)$ y se le denominó prueba $\mathrm{Pi}^{*} \mathrm{~N}^{\circ} 3$. 
Tabla 7. Grado de blanco Berger $\left({ }^{\circ} \mathrm{Be}\right)$ de los tejidos de algodón blanqueados a partir de efluentes textiles decolorados del Corazol Black B 133 \% (Black 5) y el blanco estándar.

\begin{tabular}{lcccc}
\hline Prueba & $\mathrm{L}$ & $\mathrm{C}$ & $\mathrm{h}$ & Grado de Blanco $\left({ }^{\circ} \mathrm{Be}\right)$ \\
\hline Estándar & 93,23 & 6,24 & 90,76 & 76,61 \\
$\mathrm{Pi} \mathrm{N}^{\circ} 1$ & 83,38 & 8,11 & 75,02 & 54,36 \\
$\mathrm{Pi} \mathrm{N}^{\circ} 2$ & 83,38 & 8,11 & 75,02 & 50,04 \\
$\mathrm{Pi}^{*} \mathrm{~N}^{\circ} 3$ & 92,26 & 3,39 & 95,01 & 73,41 \\
\hline
\end{tabular}

Se obtuvieron las gráficas de las curvas espectrales de reflectancia a lo largo del rango de longitud de onda del espectro visible para las pruebas iniciales (Pi) ensayadas en un volumen de $50 \mathrm{~mL}$ a partir de una dosificación inicial de Fenton, la cual no dio resultados positivos ya que persistió la coloración en el agua tratada. No se logró el grado de blanco y los resultados de capilaridad e hidrofilidad fueron mínimas en los tejidos y casi inexistentes ya que un textil al ser sometido a un proceso de blanqueo tan enérgico como éste debió presentar propiedades óptimas de capilaridad e hidrofilidad. La dosificación Fenton ajustada dio un mejor grado de blanco y un acercamiento al color del blanco estándar, esto se puede apreciar en la curva de reflectancia mostrada en la figura 7 , determinándose al tejido blanqueado prueba $\mathrm{Pi}^{*} \mathrm{~N}^{\circ} 3$ como el más próximo al blanco estándar.

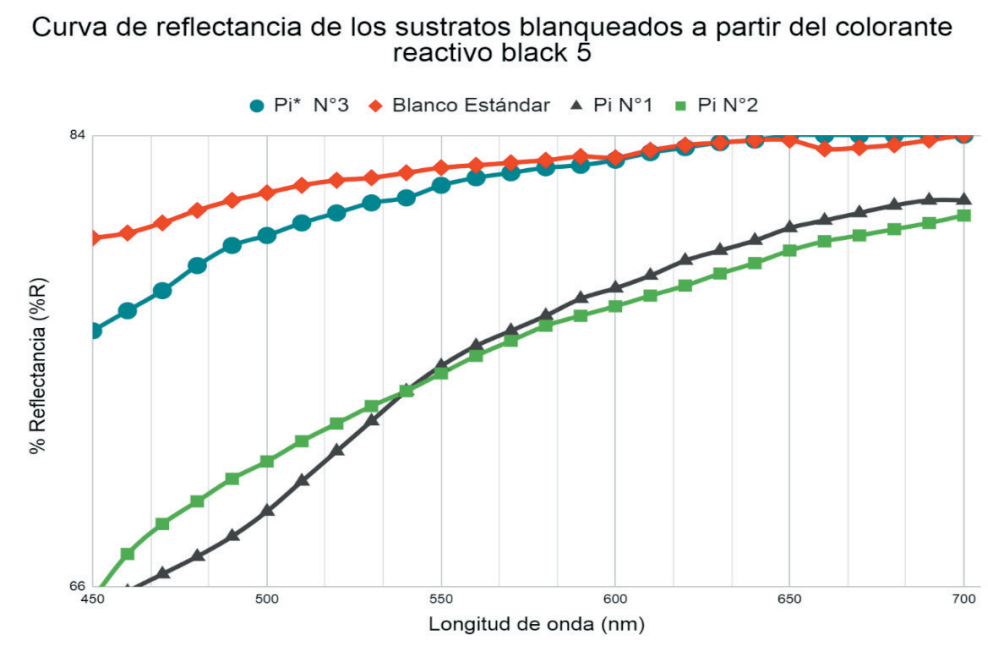

Figura 7. \% Reflectancia vs. Longitud de onda (nm) de los tejidos de algodón blanqueados a partir de efluentes decolorados del colorante reactivo Corazol Black B $133 \%$ (Black 5) y el blanco estándar.

Posterior al proceso de blanqueo a partir efluentes reales decolorados del colorante Black SD tratados con diferentes dosis de Fenton se obtuvieron sustratos textiles blanqueados mostradas en la tabla 8. La muestra textil denominada PP B es la que presenta el mejor grado de Blanco $\left(74,73^{\circ} \mathrm{Be}\right)$ y se obtuvo con el efluente tratado con dosificación Fenton ajustada de $0,0156 \mathrm{~g} / \mathrm{mL}$ según la tabla 1 para el colorante Black SD. 
Tabla 8. Grado de blanco Berger $\left({ }^{\circ} \mathrm{Be}\right)$ de los tejidos de algodón blanqueados a partir de efluentes textiles decolorados del Corafix Black SD (Black SD) y el blanco estándar.

\begin{tabular}{lcccc}
\hline Prueba & $\mathrm{L}$ & $\mathrm{C}$ & $\mathrm{h}$ & Grado de Blanco ${ }^{\circ} \mathrm{Be}$ \\
\hline Estándar & 93,23 & 6,24 & 90,76 & 76,61 \\
PPi 1 & 80,44 & 8,99 & 89,33 & 61,26 \\
PPi 2 & 90,43 & 5,24 & 85,37 & 63,09 \\
PP B & 92,93 & 6,26 & 90,78 & 74,73 \\
\hline
\end{tabular}

Finalmente, se obtuvieron las curvas espectrales de reflectancia de los blanqueos a partir de efluentes textiles reales decolorados del colorante reactivo Black SD y del blanco estándar que se muestran en la figura 8 . Se observa que la PP B es la muestra textil más parecida en color al blanco estándar.

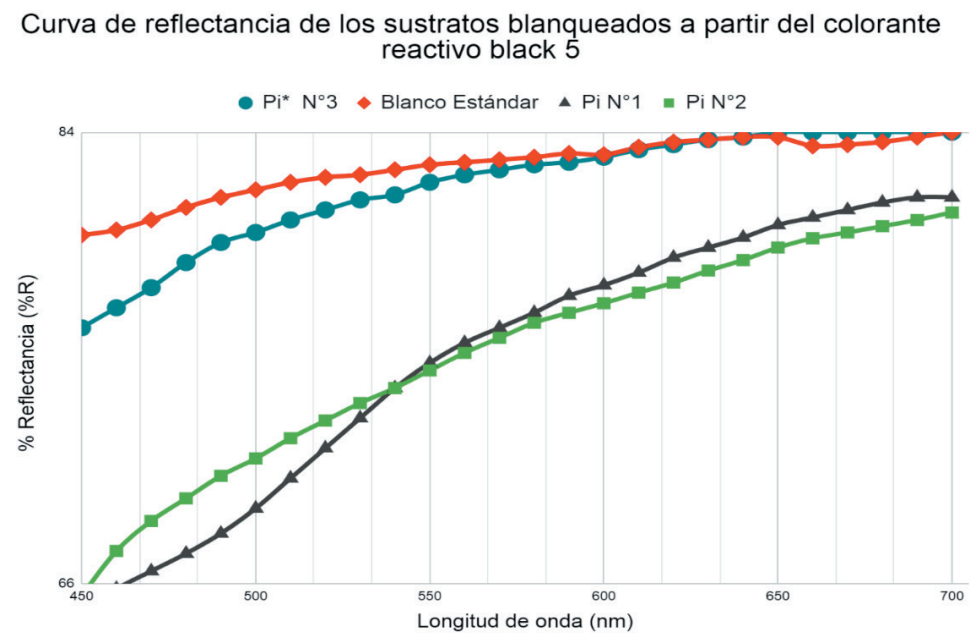

Figura 8. \% Reflectancia vs. Longitud de onda (nm) de los tejidos de algodón blanqueados a partir de efluentes decolorados del colorante reactivo Corafix Black SD (Black SD) y el blanco estándar.

\section{Evaluación de calidad de los tejidos blanqueados}

Se repitieron las pruebas de blanqueo y se realizó el control de calidad según las normas internacionales que son aplicadas en las empresas textiles peruanas para determinar otras características, incluso más importantes que el grado de blanco como: la hidrofilidad, que se aprecia en la figura 9, capilaridad, mostrada en la figura 10 y la tabla 9, que determinaron que el proceso de blanqueo fue efectivo para la remoción de impurezas, ceras y/o aceites. 


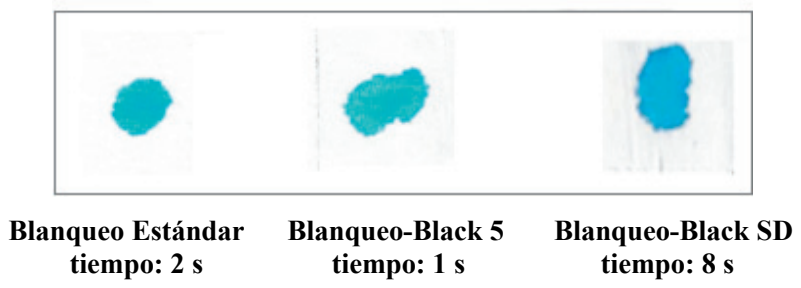

Figura 9. Resultados de la prueba de hidrofilidad (Norma AATCC 79-2014) del Blanqueo estándar vs. Blanqueos a partir de efluentes tratados de colorante reactivo Black 5 y efluentes de colorante reactivo Black SD en las pruebas con resultados óptimos.

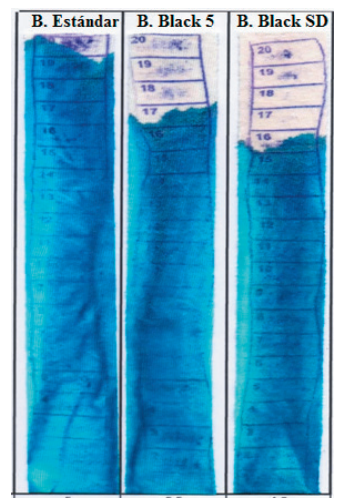

Figura 10. Capilaridad (Norma AATCC 197-2013) de la tela blanqueada estándar vs. Blanqueos con efluentes Black 5 y Black SD con resultados óptimos.

Tabla 9. Resultados de la capilaridad según Norma AATCC 197-2013 con resultados óptimos para las 3 muestras a los 5 minutos todas subieron más de $6 \mathrm{~cm}$.

\begin{tabular}{cccc}
\hline $\begin{array}{c}\text { Tiempo } \\
(\min )\end{array}$ & $\begin{array}{c}\text { Blanqueo } \\
\text { Estándar }(\mathrm{cm})\end{array}$ & $\begin{array}{c}\text { Blanqueo- } \\
\text { Black 5 }(\mathrm{cm})\end{array}$ & $\begin{array}{c}\text { Blanqueo- } \\
\text { Black SD }(\mathrm{cm})\end{array}$ \\
\hline 1 & 5,0 & 5,5 & 4,5 \\
3 & 7,5 & 7,2 & 6,2 \\
5 & 9,0 & 8,5 & 7,5 \\
30 & 19,0 & 16,5 & 16,0 \\
\hline
\end{tabular}




\section{CONCLUSIONES}

- La decoloración de los efluentes textiles reales mediante el método Fenton es una propuesta viable para tratar estos efluentes de tinturas con colorantes reactivos y su posterior reúso en procesos de blanqueo químico con características similares en capilaridad e hidrofilidad y con respecto a tono, para los dos tipos de efluentes se obtuvieron valores óptimos similares al blanqueo estándar.

- El método Fenton es eficaz para el tratamiento de estos tipos de colorantes reactivos, siendo $0,0156 \mathrm{~g} / \mathrm{mL}$ y $0,0251 \mathrm{~g} / \mathrm{mL}$ las relaciones óptimas del reactivo Fenton en un tiempo de reacción de $90 \mathrm{~min}$, con $\mathrm{pH}$ óptimos de tratamiento de 3,70 y 3,88 para los efluentes textiles provenientes de teñido con colorantes Corafix Black SD (Black SD) y Corazol Black B 133 \% (Black 5), respectivamente.

- $\quad$ El tejido celulósico blanqueado a partir de efluentes textiles reales del colorante Black 5 decolorados con el método Fenton, tuvo un tiempo de hidrofilidad de 12 segundos, capilaridad de $14 \mathrm{~cm}$ a los 30 minutos y un grado de blanco de $73,41{ }^{\circ} \mathrm{Be}$, similar al blanqueo estándar con agua blanda $\left(76,61^{\circ} \mathrm{Be}\right)$. Por lo tanto, el grado de blanco de los sustratos textiles celulósicos no se altera usando baños tratados con las dosificaciones óptimas de Fenton aún si el tratamiento no completó la mineralización del efluente.

- Asimismo, con los efluentes textiles reales del colorante Black SD se obtuvo un tejido celulósico blanqueado con propiedades similares al obtenido con los efluentes textiles reales del Black 5 .

- $\quad$ Por lo tanto, se puede inferir que un teñido a partir de estos sustratos textiles blanqueados con efluentes reales decolorados con el método Fenton darían resultados similares a teñidos a partir de sustratos blanqueados con agua blanda.

\section{AGRADECIMIENTOS}

Los autores agradecen al Instituto de Investigación de la Facultad de Ingeniería Química y Textil de la UNI por el financiamiento otorgado para el desarrollo de esta investigación. Al Ing. Javier Cárdenas, Jefe del Laboratorio de Química Aplicada de la FIQT, al Ing. Rigoberto Marín, Jefe del Laboratorio Textil $\mathrm{N}^{\circ} 24$ de la FIQT por las facilidades brindadas para la realización de los ensayos y a la empresa Coltex Perú S.A. por facilitarnos las muestras de los colorantes en estudio y los efluentes reales. 


\section{REFERENCIAS BIBLIOGRÁFICAS}

1. Sánchez CM. La industria textil, la segunda más contaminante del mundo. [Internet]. XL Semanal [citado 18 de enero 2020]. Disponible en: https:/www.xlsemanal.com/ actualidad/20160913/cataclismo-la-fast-fashion.html

2. Kolorjet Chemicals Pvt. Ltd. Textile Dyes. Dyes \& Pigments. Mumbai: Kolorjet Chemicals Pvt. Ltd. Textile Dyes; 2010.

3. Blanco Jurado J. Degradación de un efluente textil real mediante procesos Fenton y Foto-Fenton [Internet] [Tesis de máster]. Barcelona: UPC, Escola Tècnica Superior d'Enginyers de Camins, Canals i Ports de Barcelona; 2009 [citado 1 octubre 2020]. Disponible en: http://hdl.handle.net/2099.1/8325

4. Gamarra C, La Rosa-Toro A. Decoloración del anaranjado de metilo empleando el sistema Fenton. Rev Soc Quim Perú. 2014; 80: 21-34.

5. García J, Castellanos M, Uscátegui Ángela, Fernández J, Pedroza A, Daza C. Remoción de colorantes sintéticos mediante el proceso Fenton heterogéneo usando Fe2O3 soportado en carbón activado obtenido a partir de residuos de rosas. Univ Sci. [Internet] 2012; 17: 303-314. [Citado 2 octubre 2020]. Disponible en: https://revistas.javeriana. edu.co/index.php/scientarium/article/view/4035

6. Rodríguez R, Flesler F, Lehmann V. Oxidación avanzada Sistema Fenton en tratamiento de efluentes industriales de alta carga orgánica. Inti Ambiente. 2010; 1-2.

7. Nichela DA. Estudio del mecanismo y la cinética de degradación de contaminantes aromáticos. [tesis de doctorado]. La Plata: Universidad Nacional de La Plata; 2012.

8. Terán G, Posligua P, Banchón C. Dosificación mínima del reactivo Fenton para la remediación de agua contaminada con colorantes. Enfoque UTE. 2015; 6: 65-80.

9. Meric S, Kaptan D, Ölmez T. Color and COD removal from wastewater containing Reactive Black 5 using Fenton's oxidation process. Chemosphere. 2004; 54: 435-441.

10. Ribeiro K, de Andrade MT, Fujirawa ST. Preparation and application of cellulose acetate/Fe films in the degradation of Reactive Black 5 dye through photo-Fenton reaction. Environ Technol. 2016; 37: 1664-1675.

11. Clemente JP. Aplicación de procesos de oxidación avanzada (Fotocatálisis solar) para tratamiento y reutilización de efluentes textiles. [Tesis doctoral]. Valencia: Universidad Politécnica de Valencia; 2008.

12. Gutiérrez CC. Sistema Fotoquímico para el tratamiento de agua residual textil sintética con contaminantes orgánicos persistentes. [Internet] [Proyecto de Investigación]. Callao: Universidad Nacional del Callao; 2015[Citado 12 setiembre 2020]. Disponible en: http://repositorio.unac.edu.pe/handle/UNAC/1003

13. Lucas M, Peres J. Decolorization of the azo dye Reactive Black 5 by Fenton and photoFenton oxidation. Dyes Pigm. 2006; 71: 236-244. 